\title{
INFLUENCIA ADRENÉRGICA Y COLINÉRGICA SOBRE LA PRODUCCIÓN DE ESPECIES REACTIVAS DE OXÍGENO EN NEUTRÓFILOS HUMANOS*
}

\author{
Job Nexar-QH'1,2,a, Ana E. Caleiro Seixas ${ }^{3, b}$
}

\begin{abstract}
RESUMEN
Objetivos. Determinar y comparar el efecto de fármacos agonistas adrenérgicos y colinérgicos sobre la producción de especies reactivas de oxígeno (ROS) en neutrófilos de individuos sanos. Materiales y métodos. Se tomaron muestras de sangre total de cinco participantes para purificar los neutrófilos mediante el método de gelatina. Se midió la producción de ROS por quimioluminiscencia (QLM) usando un contador de centelleo y forbol-12-miristato-13-acetato (PMA) como estímulo. También se realizaron pruebas sin PMA para medir la producción espontánea. Posteriormente, con el mismo método se midió la formación de ROS en presencia de nicotina (agonista colinérgico), salbutamol y clonidina (agonistas adrenérgicos), cada uno en concentraciones de $10^{-2} \mathrm{M}, 10^{-3} \mathrm{M}, 10^{-4} \mathrm{M}$ y $10^{-5} \mathrm{M}$. Se calculó el área integrada bajo las curvas de QLM y se halló el porcentaje de inhibición o de estimulación según sea el caso. Se comparó el efecto provocado por las drogas con sus controles correspondientes y se realizó el análisis estadístico. Resultados. Se obtuvo una disminución de la producción de ROS como efecto de las sustancias estudiadas con una diferencia significativa entre los controles y el efecto producido a $10^{-2} \mathrm{M}, 10^{-3} \mathrm{M}$ y $10^{-4} \mathrm{M}$. Este efecto aumentó de intensidad conforme la concentración de las drogas se incrementó. Los mayores porcentajes de inhibición se mostraron a $10^{-2} \mathrm{M} \mathrm{y} 10^{-3} \mathrm{M}$. Salbutamol presentó los máximos valores con todas las concentraciones con diferencia significativa entre su inhibición y la generada por las demás drogas. Conclusiones. Los estímulos adrenérgico y colinérgico tienen un efecto inhibitorio de la producción de ROS en neutrófilos de individuos sanos.
\end{abstract}

Palabras clave: Neutrófilos; Agonistas Adrenérgicos; Agonistas Colinérgicos; Psiconeuroinmunología (fuente: DeCS BIREME).

\section{ADRENERGIC AND CHOLINERGIC INFLUENCE ON THE PRODUCTION OF REACTIVE OXYGEN SPECIES IN HUMAN NEUTROPHILS*}

\begin{abstract}
Objectives. To determine and compare the effect of adrenergic and cholinergic agonist drugs on the production of reactive oxygen species (ROS) in neutrophils of healthy individuals. Materials and Methods. Whole blood samples were taken from five participants to purify neutrophils using the gelatin method. The production of chemiluminescent (QLM) ROS was measured using a scintillation counter and phorbol-12-myristat-13-acetate (PMA) as a stimulus. Non-PLA tests were also conducted to measure spontaneous production. Subsequently, with the same method, ROS formation was measured in the presence of nicotine (cholinergic agonist), salbutamol, and clonidine (adrenergic agonists), each in concentrations of $10^{-2} \mathrm{M}, 10^{-3} \mathrm{M}, 10^{-4} \mathrm{M}$, and $10^{-5} \mathrm{M}$. The area integrated under the QLM curves was calculated and the percentage of inhibition or stimulation was found as the case may be. The effect of the drugs was compared with their corresponding controls and statistical analysis was carried out. Results. A decrease in the production of ROS was obtained as an effect of the substances studied with a significant difference between the controls and the effect produced at $10^{-2} \mathrm{M}, 10^{-3} \mathrm{M}$, and $10^{-4} \mathrm{M}$. This effect increased in intensity as drug concentration increased. The highest percentages of inhibition were shown at $10^{-2} \mathrm{M}$ and $10^{-3} \mathrm{M}$. Salbutamol presented the maximum values with all the concentrations with a significant difference between its inhibition and that generated by the other drugs. Conclusions. Adrenergic and cholinergic stimuli have an inhibitory effect on the production of ROS in neutrophils of healthy individuals.
\end{abstract}

Keywords: Neutrophils; Adrenergic agonists; Cholinergic agonists; Psychoneuroimmunology (source: MeSH NLM).

Universidad Nacional de San Agustín. Arequipa, Perú.

Neurologic Clinic and Policlinic of the University Hospital Basel, University of Basel, Suiza.

Departamento de Física y Química, Facultad de Ciencias Farmacéuticas de Ribeirão Preto, Universidad de São Paulo. Ribeirão Preto, Brasil.

Médico cirujano; ${ }^{\mathrm{b}}$ químico farmacéutico.

El artículo es parte de la tesis de pregrado de Job Nexar Quispe Huamán para obtener el grado de médico cirujano de la Universidad Nacional San Agustín.

Recibido: 31/10/2018 Aprobado: 27/02/2019 En línea: 21/03/2019

Citar como: Nexar-QH J, Caleiro Seixas AE. Influencia adrenérgica y colinérgica sobre la producción de especies reactivas de oxígeno en neutrófilos humanos. Rev Peru Med Exp Salud Publica. 2019;36(1):54-61. doi:10.17843/rpmesp.2019.361.4026. 


\section{INTRODUCCIÓN}

Según la Organización Mundial de la Salud, los trastornos psiquiátricos se encuentran dentro de las primeras causas de morbilidad y consecuente incapacidad a nivel mundial ${ }^{(1)}$, y dentro de ellos la ansiedad y depresión son los más frecuentes en muchos países ${ }^{(2)}$. Datos epidemiológicos en Latinoamérica muestran que la situación es similar a la de Norteamérica, Europa y naciones de otras áreas ${ }^{(3)}$, siendo así un serio problema de salud pública internacional. Esta situación podría estar provocando diversas consecuencias sobre la salud de las personas, pues es conocido que los factores psicológicos pueden alterar la respuesta inmunológica de nuestro organismo ${ }^{(3,4)}$.

Algunos estudios reportan que esta alteración obedece a cambios observados en el adecuado funcionamiento del sistema inmunológico, tanto en su componente celular como humoral ${ }^{(5,6)}$. El campo de la psiconeuroinmunología sostiene que estos cambios se producen porque, además de la conocida integración neuro-endocrino-inmunológica, el sistema nervioso central ejerce una influencia directa sobre el sistema inmunológico y se establece una comunicación bidireccional con este (7). Se propone que ciertos neurotransmisores constituyen los mediadores químicos que permiten la interacción de estos dos sistemas ${ }^{(8)}$. Incluso ha podido identificar la existencia de receptores específicos en linfocitos y macrófagos ${ }^{(9)}$.

Recientemente se viene estudiando también al sistema nervioso autónomo como regulador, a través de la liberación de catecolaminas y acetilcolina ${ }^{(10)}$. Se ha demostrado que estos neurotransmisores actúan sobre receptores específicos en leucocitos de la inmunidad adaptativa ${ }^{(10)}$ y se ha señalado a la descarga colinérgica del nervio vago como una de las vías principales y más importantes de esta regulación directa ${ }^{(11,12)}$. Sin embargo, no se ha tomado muy en cuenta a las células de la inmunidad innata, especificamente los neutrófilos que constituyen la primera línea de defensa inmunológica y son importantes en el proceso inflamatorio. Podría ser que también respondan a dichos neurotransmisores y que, por ende, estén bajo la misma regulación autonómica, pero se requieren de estudios que demuestren estos efectos.

Por ello, se estudiaron neutrófilos humanos in vitro, evaluando su producción de especies reactivas de oxígeno (ROS) por ser este un componente central en su actividad microbicida. El objetivo fue determinar y comparar el efecto producido ante el estímulo adrenérgico y colinérgico aplicado in vitro.

\section{MATERIALES Y MÉTODOS}

\section{DISEÑO DEL ESTUDIO}

Se realizó un estudio experimental con neutrófilos de muestras de sangre total procedentes de cinco voluntarios

\section{MENSAJES CLAVE}

Motivación para realizar el estudio. Un mal estado emocional y psicológico puede influir negativamente en el sistema inmunológico de las personas. Lo puede hacer de distintas formas y con resultados apreciables.

Principales hallazgos. En el presente estudio se encontró una reducción de la funcionalidad de un tipo de defensas de cuerpo, después de verse sometidos a ciertas sustancias activadoras, cuya liberación esta aumentada durante estados de ansiedad y depresión.

Implicancias.Los resultados resaltan la importancia de promover y mantener una buena salud emocional a fin de prevenir la alteración del desempeño de células inmunitarias en nuestro organismo.

clínicamente sanos al momento del estudio con una edad comprendida entre 18 y 45 años, sexo masculino y hemograma con recuento porcentual y total de leucocitos dentro de valores normales. Ninguno de los voluntarios tuvo antecedente de tratamiento antidepresivo o ansiolítico, consumo de alcohol, tabaco, drogas o medicamentos inmunomoduladores.

\section{PROCEDIMIENTO EXPERIMENTAL}

De cada sujeto, se extrajeron $20 \mathrm{~mL}$ de sangre por punción venosa. Los neutrófilos fueron purificados por el método de la gelatina, descrito por Lucisano y Mantovani ${ }^{(13)} y$ con algunas modificaciones de Kabeya ${ }^{(14)}$ para obtener un concentrado de neutrófilos. La sangre colectada fue diluida volumen a volumen en solución Alséver ( $\mathrm{pH} 6,1)$ utilizada como anticoagulante y posteriormente fue centrifugada. Después se removió el plasma y la camada de células mononucleares por aspiración con pipeta. El sedimento fue diluido en solución de gelatina a $2,5 \%$ e incubado en baño maría a $37^{\circ} \mathrm{C}$ por 15 minutos. El sobrenadante rico en neutrófilos fue diluido en igual volumen de $\mathrm{CINa} 0,15 \mathrm{~mol} / \mathrm{L}$ y centrifugado. Para lisar los eritrocitos remanentes, el sedimento fue suspendido en solución de $\mathrm{CINH} 4$ 0,83\% (pH 7,2) e incubado por cinco minutos a $37^{\circ} \mathrm{C}$, luego se centrifugó nuevamente. El sedimento fue lavado con $\mathrm{CINa} 0,15 \mathrm{~mol} / \mathrm{L}$ y suspendido en $1 \mathrm{~mL}$ de solución Hanks conteniendo $0,1 \%$ de gelatina, obteniendo así un concentrado de neutrófilos.

Se coloreó $10 \mu \mathrm{L}$ del concentrado con $990 \mathrm{~mL}$ de líquido de Turk y se contabilizó en una cámara Neubauer. De acuerdo al número de células calculado, el concentrado fue diluido con Solución Hanks conteniendo $0,1 \%$ de gelatina hasta obtener una concentración de $4 \times 10^{6}$ células $/ \mathrm{mL}$, que es la cantidad patrón con la que se trabajó en todos los procedimientos. La viabilidad celular fue evaluada por el ensayo de colorante azul de tripan. Las preparaciones de neutrófilos obtenidas presentaron cerca del $90 \%$ de viabilidad y de pureza, siendo linfocitos y algunos monocitos los principales contaminantes. 
Se usó forbol-12-miristato-13-acetato (PMA) para estimular la producción de ROS en los neutrófilos. El PMA fue disuelto en dimetilsulfóxido (DMSO) en concentración de $10^{-2} \mathrm{~mol} / \mathrm{L}$. Se utilizó luminol a $100 \mu \mathrm{mol} / \mathrm{L}$ en solución Hanks para la amplificación de la respuesta en la medición de quimioluminiscencia (QLM).

La acetilcolina, adrenalina y noradrenalina tienen un tiempo de vida media demasiado corto, por ello, se utilizaron drogas que actúan en los mismos receptores para dichas sustancias, dichas drogas fueron: nicotina ((-)-Nicotine hydrogen tartrate-SIGMA), agonista de receptores colinérgicos tipo nicotínico), salbutamol (salbutamol S8260-100MG-SIGMA, agonista de receptores adrenérgicos tipo beta 2) y clonidina (clonidine hydrochloride C7897-100MG- SIGMA, agonista de receptores adrenérgicos tipo alfa 2) con las cuales se preparó soluciones acuosas a cuatro concentraciones $\left(10^{-2} \mathrm{M}, 10^{-3} \mathrm{M}, 10^{-4} \mathrm{M}\right.$, y $\left.10^{-5} \mathrm{M}\right)$.

Las muestras de neutrófilos fueron utilizadas inmediatamente después de su obtención y se mantuvieron refrigeradas a $4{ }^{\circ} \mathrm{C}$ durante todo el tiempo de duración de los experimentos, con la finalidad de preservar su viabilidad. Se siguió la metodología descrita por Andrade et al. ${ }^{(15)}$ para medir la producción de ROS por QLM. En placas blancas de 96 pozos se adicionó: $50 \mu \mathrm{L}$ del preparado de neutrófilos, $30 \mu \mathrm{L}$ de luminol y $100 \mu \mathrm{L}$ de solución Hanks (en el caso de los grupos control) o $100 \mu \mathrm{L}$ de solución de las drogas en las concentraciones establecidas (en el caso de los grupos experimentales); en seguida fue adicionado $20 \mu \mathrm{L}$ de PMA $10^{-2} \mathrm{~mol} / \mathrm{L}$ hasta llegar a la concentración de $10^{-7} \mathrm{~mol} / \mathrm{L}$ e inmediatamente se midió la QLM, en fotones contados por segundo, durante 15 minutos a $37{ }^{\circ} \mathrm{C}$ en luminómetro de microplacas. Además se realizaron simultáneamente experimentos «blanco» 0 «basal»; para ello, se procesaron muestras sin PMA, siendo reemplazado por $20 \mu \mathrm{L}$ de solución Hanks, para evaluar la producción espontánea de ROS. Cada experimento tuvo su propio control y basal. A partir de los perfiles cinéticos de QLM en función del tiempo, fueron calculados los valores de área integrada (área bajo la curva) de 0 a 15 minutos. Estos valores representaron la cantidad total de ROS producidos en ese intervalo de tiempo.

Posteriormente, se realizó el estudio de la viabilidad celular ante el efecto de las drogas estudiadas. Para ello se incubaron, durante 15 minutos, muestras de neutrófilos con soluciones de las drogas en las mismas concentraciones utilizadas en los experimentos, para luego proceder con el ensayo de colorante azul de tripan.

\section{ANÁLISIS DE DATOS}

Se realizaron tres experimentos con cada concentración de las drogas en cada participante, haciendo un total de 15 repeticiones por cada concentración de nicotina, salbutamol y clonidina. De igual forma 15 repeticiones control y basal.
Se calcularon los valores de área integrada de las curvas formadas por las drogas a diferentes concentraciones, para luego realizar el análisis estadístico. Estos valores se compararon con los controles correspondientes para evaluar cambios en la cantidad producida de ROS provocada por las sustancias analizadas y se determinó el porcentaje de inhibición o estimulación, según sea el caso, en relación a los respectivos controles, siguiéndose la siguiente fórmula:

$\% I=100-[(A S / A C) \times 100] \quad \% E=[(A S / A C) \times 100]-100$

Donde:

$\%$ I: porcentaje de inhibición en relación al control $\% \mathrm{E}$ : porcentaje de estimulación en relación al control

AS: área integrada de las curvas en cada concentración.

$\mathrm{AC}$ : área integrada de las curvas control

El procesamiento y análisis de los datos se hizo con el software GraphPad Prism versión 5.0 para Windows. Los resultados fueron expresados como media y desviación estándar. Se aplicó el test de Shapiro-Wilk para determinar la distribución normal de la muestra y luego los resultados fueron comparados estadísticamente por el método de análisis de varianza (ANOVA) seguido por el test de Dunnett. Los valores de $p<0,05$ fueron considerados significativos.

\section{ASPECTOS ÉTICOS}

La participación del estudio fue voluntaria previa firma de consentimiento informado y se obtuvo la aprobación por el Comité de Ética de la Facultad de Ciencias Farmacéuticas de Ribeirão Preto de la Universidad de São Paulo (FCFRP. USP. CEP/FCFRP 331).

\section{RESULTADOS}

En todas las mediciones control se detectó un aumento progresivo en la producción de ROS conforme se avanzaba del minuto cero hasta el minuto 15 . Se observó también un pico máximo que fue alrededor de los 500 segundos después del inicio, luego de lo cual se obtuvo una gradual disminución. En las mediciones con los grupos basal se detectó una mínima emisión, la cual fue aumentando leve y gradualmente pero manteniéndose siempre a niveles muy bajos.

El efecto de la nicotina varió de acuerdo a la concentración de la droga, habiendo una disminución de la producción de ROS en comparación con el grupo control, al usar dosis elevadas. La dosis más alta, es decir $10^{-2} \mathrm{M}$, provocó una disminución casi total, incluso por debajo del nivel medido en el grupo basal. Cuando la concentración de nicotina fue disminuida a $10^{-3} \mathrm{M}$ y $10^{-4} \mathrm{M}$ se detectó una pérdida de este efecto, aunque las mediciones continuaban siendo inferiores al control en todo momento. Ocurrió algo distinto con la concentración $10^{-5} \mathrm{M}$ ya que provocó un ligero aumento con un pico máximo que tiende a ser levemente superior al pico del control (Figura 1). El área integrada de las curvas generadas por las dos concentraciones más 


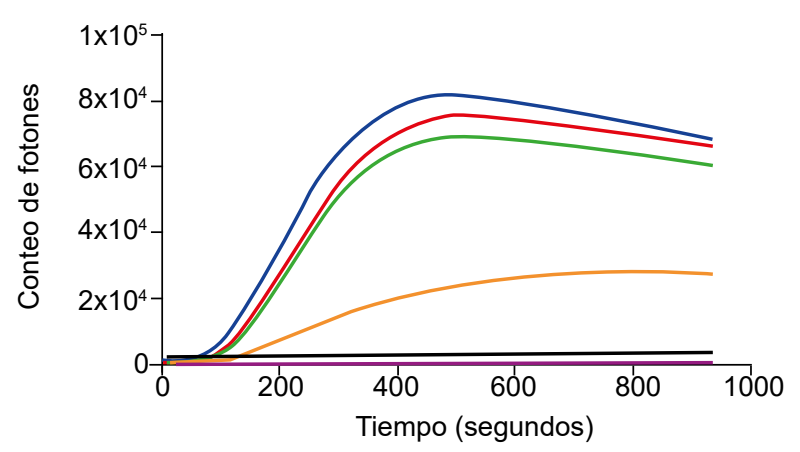

$\begin{array}{ll}\text { - Basal } & -10^{-2} \mathrm{M}+\text { PMA } \\ \text { - PMA } & -10^{-4} \mathrm{M}+\mathrm{PMA} \\ -10^{-3} \mathrm{M}+\mathrm{PMA} & -10^{-5} \mathrm{M}+\mathrm{PMA}\end{array}$

PMA: forbol-12-miristato-13-acetato

Figura 1. Curvas de quimioluminiscencia bajo el efecto de nicotina.

altas resultó ser significativo $(p<0,05)$ al área integrada de la curva control (Figura 2).

En el caso del salbutamol también la concentración máxima de la droga ocasionó una caída de la emisión de fotones hasta valores muy ínfimos y el efecto fue perdiéndose conforme se bajaba la concentración (Figura 3). Se hallaron diferencias entre el área integrada de la curva control y las áreas de las curvas generadas por las concentraciones mayores (Figura 4). Con la clonidina, las concentraciones de $10^{-2} \mathrm{M}$ y $10^{-3} \mathrm{M}$ obtuvieron curvas menores que el control (Figura 5) y el área integrada de dichas curvas también resultó ser significativamente diferente al compararlas con el control respectivo (Figura 6).

Se calculó el porcentaje de inhibición provocado por las drogas a una misma concentración y luego se comparó entre ellas para determinar aquellas con el mayor efecto. Se halló que a $10^{-2} \mathrm{M}$ la nicotina tiene el mayor efecto, con casi $100 \%$ de inhibición. El salbutamol inhibió $98,1 \%$, un porcentaje muy cercano al anterior por lo que no se halló diferencia estadísticamente significativa. En cambio, la clonidina sí tuvo un porcentaje de inhibición notablemente menor en comparación con las anteriores drogas. Cuando la concentración se baja a $10^{-3} \mathrm{M}$, pudo apreciarse una diferencia significativa entre los porcentajes de inhibición generados por cada droga, siendo el salbutamol el que tiene el mayor. Por último, cuando se trabajó a $10^{-4} \mathrm{M}$ los porcentajes de inhibición de la nicotina y clonidina son bajos, resaltando la clonidina que únicamente tiene un $5,7 \%$, mientras que el salbutamol inhibió más del $40 \%$; todas las diferencias fueron estadísticamente significativas en este último caso.

El post ensayo con azul de tripan mostró que la viabilidad celular de los neutrófilos, ante nicotina y clonidina en solución, se mantiene por encima del $90 \%$ en el rango de $10^{-5} \mathrm{M}$ a $10^{-3} \mathrm{M}$. Mientras que a $10^{-2} \mathrm{M}$ se reduce a un $68 \%$. Los neutrófilos preservaron su viabilidad de cerca al $90 \%$ en el caso de las soluciones con salbutamol a $10^{-5} \mathrm{M}$ y $10^{-4}$ $\mathrm{M}$. Pero hubo una reducción a un $82 \%$ y $60 \%$ en los casos de $10^{-3} \mathrm{M}$ y $10^{-2} \mathrm{M}$ respectivamente.

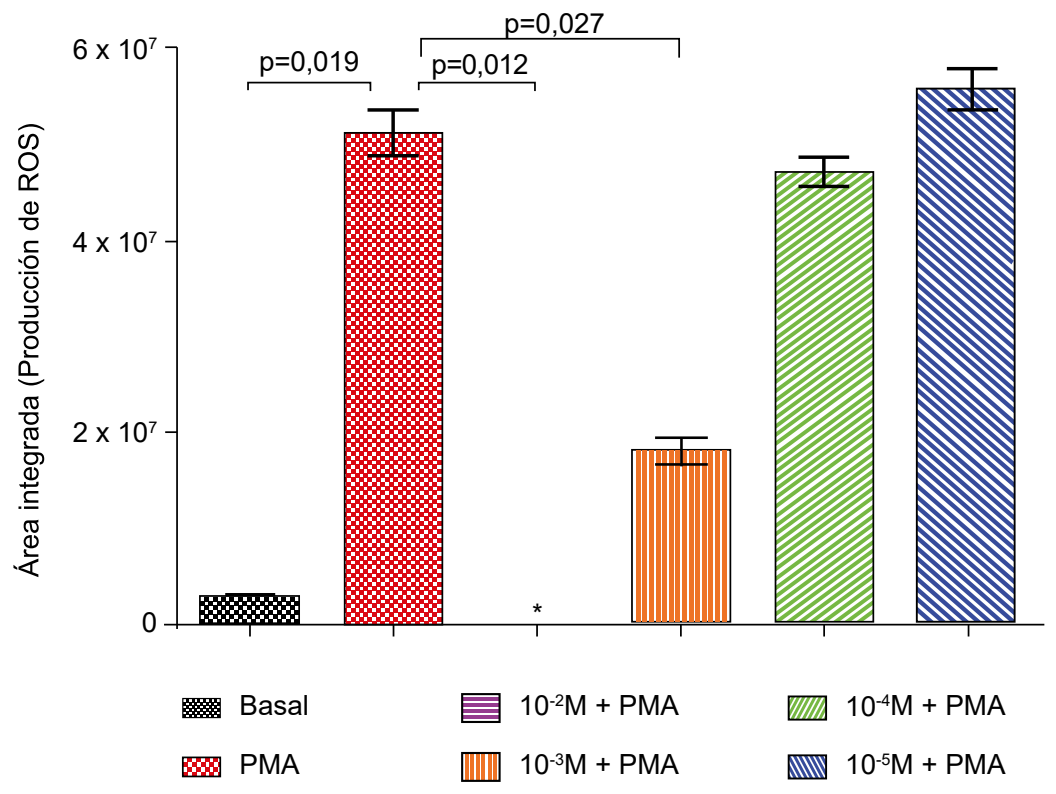

PMA: forbol-12-miristato-13-acetato

Figura 2. Área integrada de las curvas de quimioluminiscencia bajo el efecto de nicotina.

* Valor del área cercano al cero. 


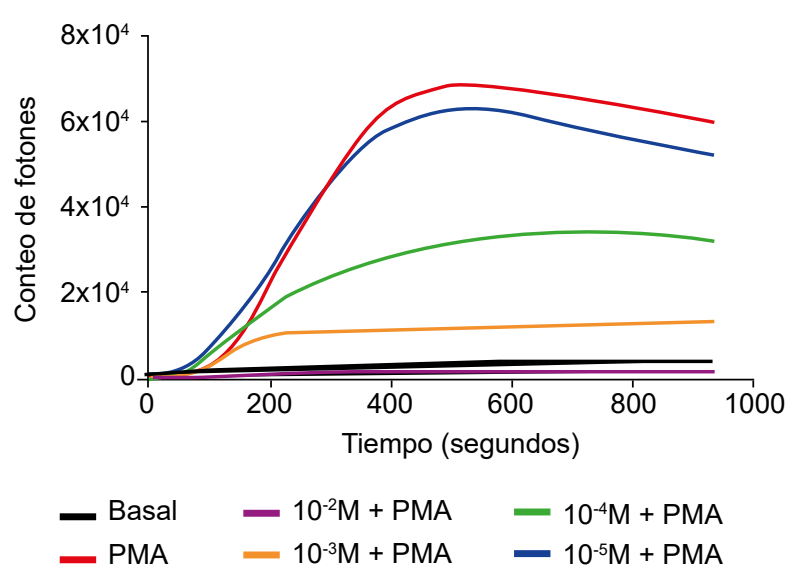

PMA: forbol-12-miristato-13-acetato

Figura 3. Curvas de quimioluminiscencia bajo el efecto de salbutamol.

\section{DISCUSIÓN}

Como la magnitud de la emisión de fotones en la prueba de QLM depende directamente de la cantidad de ROS producidas por los neutrófilos entonces se puede señalar que la Nicotina influyó en la producción de estos. A una concentración muy baja no se evidenció un descenso sino más bien una ligera tendencia al aumento que fue no significativo; sin embargo, conforme se incrementa progresivamente la concentración de nicotina, se ve que la producción de ROS se reduce considerablemente, llegando a ser prácticamente nula con la máxima concentración usada.
La inhibición fue estadísticamente significativa con las dos mayores concentraciones. Sin embargo, se evidenció una reducción importante de la viabilidad con nicotina a $10^{-2} \mathrm{M}$ en el post ensayo con azul de tripan, por lo que es posible que un efecto citotóxico provocara esa caída en la formación de ROS. No sería ese el caso con las demás concentraciones en donde la viabilidad se mantuvo por encima de $90 \%$ y por tanto la inhibición significativa a $10^{-3} \mathrm{M}$ puede ser correctamente atribuida a la nicotina.

Se utilizó nicotina en lugar de acetilcolina debido a su mayor estabilidad en solución; sin embargo, la nicotina es una droga agonista de receptores nicotínicos del sistema colinérgico y desencadena efectos semejantes a los de la acetilcolina cuando se une a estos receptores. Por ello, los neutrófilos presentarían una respuesta colinérgica que resulta en una inhibición en la producción de ROS. La existencia de esta respuesta revela la posibilidad de que los neutrófilos tengan receptores nicotínicos para acetilcolina.

Sasakawa, et al ${ }^{(16)}$ realizó un estudio similar en el rango $10^{-5} \mathrm{M}$ hasta $5 \times 10^{-4} \mathrm{M}$. Demostró también una inhibición de la producción de ROS dosis dependiente provocada por nicotina. En su estudio posterior ${ }^{(17)}$ probó que la producción de anión superóxido $\left(\mathrm{O}^{-2}\right)$ se ve afectada. En dichos estudios se trabajó también con drogas antagonistas tales como atropina y hexamethonium, las cuales no tuvieron ningún efecto significativo en la formación de ROS ni tampoco un efecto competitivo con la nicotina. Por ello, propone que la nicotina tendría un efecto directo en los neutrófilos que no es mediado por receptores nicotínicos, por lo tanto no

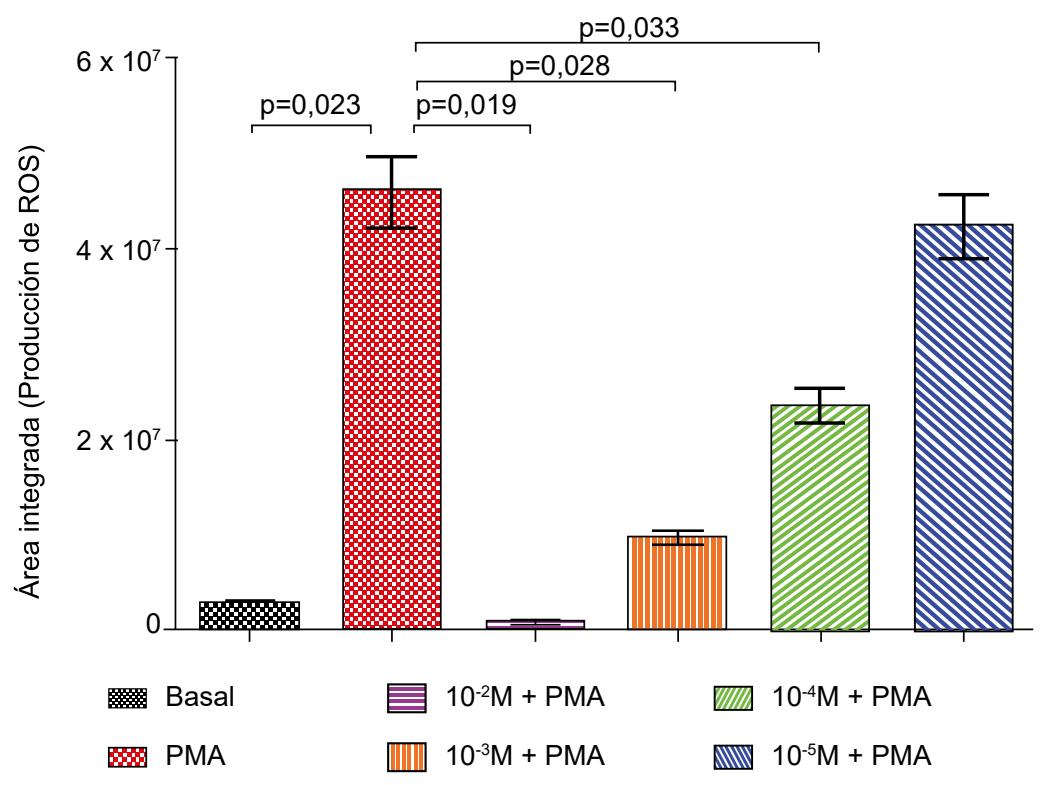

PMA: forbol-12-miristato-13-acetato

Figura 4. Área integrada de las curvas de quimioluminiscencia bajo el efecto de salbutamol. 


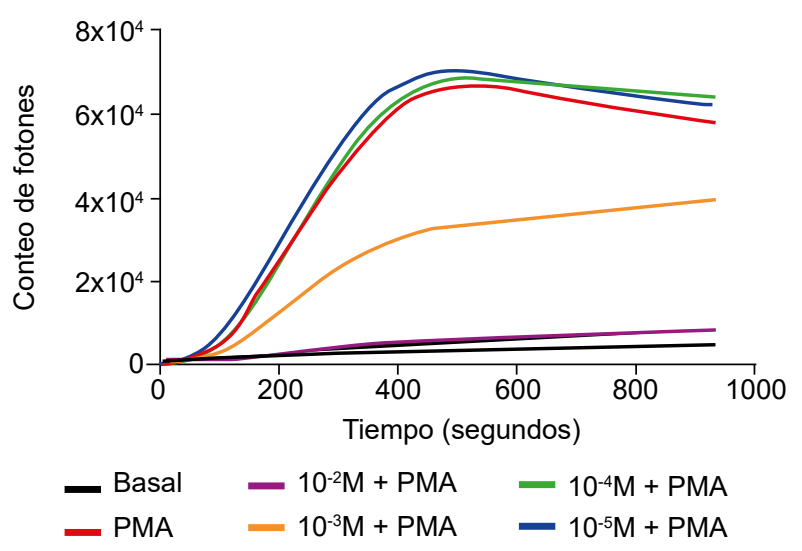

PMA: forbol-12-miristato-13-acetato

Figura 5. Curvas de quimioluminiscencia bajo el efecto de clonidina.

existiría una tal respuesta colinérgica. Sin embargo, no se han hecho estudios de expresión de genes en neutrófilos que codifiquen para estos receptores, por lo que afirmarlos o negarlos es algo que queda en discusión.

Bernik T ${ }^{(18)}$ y Tracey, et al. ${ }^{(19)}$ hablan también de la existencia de receptores para neurotransmisores inhibitorios en células de la inmunidad innata, tales como macrófagos y células dendríticas, que responden disminuyendo la producción de citoquinas proinflamatorias. Dicho estudio indica que ello permitiría un control del proceso inflamatorio en el tejido periférico y así evitar un daño tisular. En el caso de los neutrófilos el objetivo de la presencia de esta respuesta inhibitoria podría ser la misma. Aunque esta regulación tiene como propósito evitar una excesiva respuesta y daño tisular, el aumento exagerado de la estimulación de receptores nicotínicos en leucocitos podría llevar a una inhibición excesiva. Ello se demostraría con los resultados de este estudio, donde se aprecia que el incremento progresivo en la concentración de nicotina provoca una reducción en la producción de ROS. Este es sólo un estudio in vitro y son necesarios estudios clínicos, pero se puede sospechar que el uso exagerado de medicamentos semejantes, exposición a sustancias que activen los mismos receptores (como la nicotina del tabaco) o las actividades, situaciones y hábitos que incrementen la descarga colinérgica sobre neutrófilos pueden atenuar su actividad microbicida y afectar la respuesta ante una noxa.

En el presente estudio, se utilizó salbutamol, un agonista beta 2 adrenérgico, y clonidina, un agonista alfa 2 adrenérgico. A $10^{-2} \mathrm{M}$ y $10^{-3} \mathrm{M}$ ambos inhibieron significativamente la producción de ROS. El salbutamol lo hizo inclusive hasta $10^{-4} \mathrm{M}$. Sin embargo, las soluciones de mayor concentración $\left(10^{-2} \mathrm{M}\right)$ disminuyeron la viabilidad a menos del $80 \%$ según el post ensayo con azul de tripan, por lo que únicamente en este caso no se descarta un posible efecto citotóxico de las drogas agonistas que puede confundirse con un real efecto inhibitorio atribuible a los agonistas.

La manifestación de este efecto inhibitorio sugiere que los neutrófilos humanos tienen además receptores adrenérgicos específicos que median respuestas inhibitorias. Seifert, et al. ${ }^{(20)}$ menciona la existencia de receptores beta 2-adrenérgicos en neutrófilos y además indica que son receptores ligados a

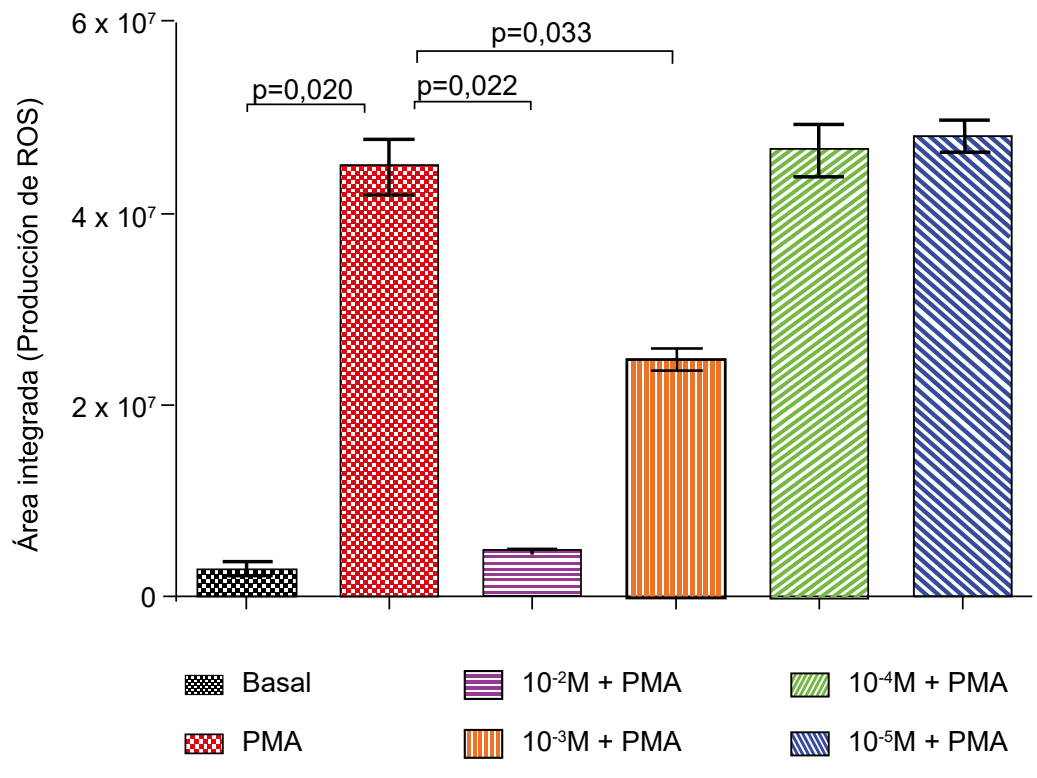

PMA: forbol-12-miristato-13-acetato

Figura 6. Área integrada de las curvas de quimioluminiscencia bajo el efecto de clonidina. 
proteínas $\mathrm{G}$, que actuarían inhibiendo la formación de anión superóxido pero en una vía independiente de cAMP. Se han realizado estudios de perfiles farmacológicos y de unión de ligandos que demuestran la presencia de estos receptores también en linfocitos y el efecto hallado fue de inhibición de reacciones inmunes celulares ${ }^{(10)}$.

Variaciones en la concentración de salbutamol produjeron cambios en la magnitud de la respuesta, la cual se incrementa en relación directa. Sus porcentajes de inhibición fueron los más altos para cada caso en comparación con las otras sustancias e inhibió significativamente la producción de ROS hasta la concentración de $10^{-4} \mathrm{M}$. Lo anterior tiene relación con varios estudios en inmunidad celular que también trabajaron con agonistas beta 2 adrenérgicos. Estos concluyen que la activación de sus receptores en neutrófilos provoca una fuerte acción supresora en la producción de ROS ${ }^{21)}$. Además, estos agonistas también pueden actuar en otras células de la inmunidad innata como macrófagos ${ }^{(22)}$ y células asesinas naturales ${ }^{(23)}$.

Con clonidina se observó un cambio de respuesta dependiente de la concentración. Hubo una tendencia al aumento con las concentraciones más bajas pero no resultó estadísticamente significativo, luego de lo cual se redujo la formación de ROS hasta más del $50 \%$. En un estudio similar realizado por Kahoru Nishina ${ }^{(24)}$, se concluye que la clonidina no tiene efecto sobre la producción de ROS, por tanto no tendrían receptores alfa adrenérgicos; sin embargo, en esa ocasión se utilizaron dosis muchísimo más bajas en el rango de $10^{-9} \mathrm{M}$, lo que podría explicar la diferencia con nuestro estudio. No obstante, no se encontró mayor bibliografía en referencia a neutrófilos y agonistas alfa adrenérgicos, pero hay estudios que muestran que macrófagos también responden y disminuyen su producción de citocinas ${ }^{(10)}$ y además Xiao et al. ${ }^{(25)}$ ha demostrado la existencia de receptores alfa 1 y 2 en células asesinas naturales, por medio de un estudio de expresión génica.

La nicotinamida adenina dinucleótido fosfato (NADPH) oxidasa cumple un papel central en el mecanismo de producción de EROs en los neutrófilos; es posible que tras la activación de receptores nicotínicos-colinérgicos o adrenérgicos, se haya inhibido esta enzima. Puede que se vean involucradas las proteínas Phox: p47phox, p67phox y p40phox, cuya translocación permite la activación de la NADPH oxidasa. Determinar los posibles mecanismos implicados y los ROS más afectados será motivo de posteriores trabajos. Se ha demostrado que las células inmunes también tienen receptores para muchos otros tipos de neurotransmisores, como: dopamina ${ }^{(26)}$ y serotonina ${ }^{(27)}$. Pero además, recientemente se ha descubierto que algunos tipos de células de la inmunidad adaptativa pueden sintetizar y liberar estos neurotransmisores así como también acetilcolina y adrenalina/noradrenalina ${ }^{(28)}$. Determinar si los neutrófilos y otras células de la inmunidad innata también poseen esta capacidad debe ser objetivo de futuros estudios.

La implicancia clínica de estos resultados reluce al pensar las posibles consecuencias de tratamientos largos con drogas agonistas de receptores beta, por ejemplo en el tratamiento inhalatorio contra el asma bronquial en donde se utilizan drogas de vida media larga. Un tratamiento largo y a dosis altas podría afectar a capacidad microbicida de los neutrófilos, aunque para demostrarlo se deben realizar estudios clínicos prospectivos y retrospectivos. Los resultados también ayudan a entender cómo la descarga simpática constante e incrementada, presente en personas con síndromes de ansiedad aguda y crónica, puede afectar la respuesta de células inmunitarias (en este caso neutrófilos) ante agentes agresores.

Como limitaciones del estudio hay que mencionar que no se pudo trabajar en intervalos de concentración más pequeños, lo cual ayudaría a una mejor descripción del fenómeno. Además, usar soluciones menos concentradas habría permitido contrastar los resultados con los de otros estudios. Por otro lado, no se pudo experimentar al mismo tiempo con drogas agonistas y antagonistas, lo que no permite identificar la existencia de algún fenómeno de competición que aumente indicios de la existencia de receptores adrenérgicos y nicotínicos en neutrófilos.

En conclusión, los estímulos adrenérgico y colinérgico provocan un efecto inhibitorio de neutrófilos humanos, que se expresa como una disminución en su producción de ROS.

Agradecimientos: A la Prof. Dra. Yara Lucisano Valim de la Facultad de Ciencias Farmacéuticas de Ribeirão Preto de la Universidad de São Paulo, por dar el permiso y las facilidades para desarrollar el presente trabajo en las instalaciones de su centro de investigación. A la Dra. Irmia Paz de la Facultad de Medicina de la Universidad Nacional San Agustín y a Alexandre Kanashiro de la Facultad de Medicina de Ribeirão Preto de la Universidad de São Paulo por el asesoramiento brindado.

Contribuciones de los autores: JQ participó en la concepción, diseño del estudio y en la redacción del artículo. JQ y $A C$ participaron en la recolección de datos, análisis e interpretación de resultados, revisión crítica y aprobación de la versión final del manuscrito.

Fuentes de financiamiento: autofinanciado.

Conflictos de interés: no existen conflictos de interés.

\section{REFERENCIAS BIBLIOGRÁFICAS}

1. Kastrup MC, Ramos AB. Global mental health. Dan Med Bull. 2007;54(1):42-3.

2. Epidemiologia de los trastornos mentales en América Latina y el Caribe. En:
Rodriguez Jorge J, Khon Robert, Levav Itzbak. Salud Mental en la Comunidad. 23th ed. Washington DC: Organización Panamericana de la Salud; 2009. p. 27-37.
3. Leserman J, Petitto JM, Golden RN, Gaynes BN, Gu H, Perkins DO, et al. Impact of stressful life events, depression, social support, coping, and cortisol on 
progression to AIDS. Am J Psychiatry. 2000;157(8):1221-8. doi: 10.1176/appi. ajp.157.8.1221.

4. Camps C, Sánchez PT, Sirera R. Inmunología, estrés, depresión y cáncer. Psicooncología. 2006;3(1):35-48.

5. Herbert TB, Cohen S.. Depression and immunity: a metha-analytic review. Psychol Bull. 1993;113(3):472-86.

6. Khansari DN, Murgo AJ, Faith RE. Effects of stress on the immune system. Immunol Today. 1990;11(5):170-5.

7. Larocca L, Calafat M, Roca V, Ramhorst R, Pérez C. El lenguaje químico de la interacción inmune-neuroendócrina. Revista QuímicaViva. 2008;7(3):197-212.

8. Vergara U. Sistema Nervioso Central y Regulación de la Respuesta Inmune. Avances en Ciencias Veterinarias. 2011;26(1-2):41-52.

9. Solano RL, Velásquez VE. Efecto inmunomodulador del estrés psicológico. Salus. 2012;16(1):51-57.

10. Caballero C, Reyes S, Guerra T, Rodríguez C, Tamez P, Weber RJ, et al. Regulación neuroendocrina del sistema inmune. Ciencia UANL. 2011;4(2):205-14.

11. Rosas-Ballina M, Olofsson PS, OchaniM, Valdes-Ferrer SI, Levine YA, Reardon C, et al. Acetylcholine-Synthesizing T Cells Relay Neural Signals in a Vagus Nerve Circuit. Science. 2011; 334(6052):98101. doi: 10.1126/science.1209985.

12. Wong $\mathrm{CH}$, Jenne CN, Lee WY, Leger C, Kubes P. Functional Innervation of Hepatic iNKT cells Is Immunosuppressive Following Stroke. Science. 2011;334(6052):101-5. doi: 10.1126/ science.1210301.

13. Lucisano YM, Mantovani B. Lysosomal enzyme release from polymorphonuclear leukocytes induced by immune complexes of $\operatorname{IgM}$ and of $\operatorname{IgG}$. J Immunology. 1984;132(4): 2015-20.

14. Kabeya LM, Kanashiro A, Azzolini AE, Soriani FM, Lopes JL, Lucisano-Valim
YM. Inhibitory effect of eight simple coumarins on the lucigenin enhanced chemiluminescence of rabbit neutrophils. Res Commun Mol Pathol Pharmacol. 2002;111(1-4):103-13.

15. Andrade MF, Kabeya LM, Azzolini AECS, Santos EOL, Figueiredo-Rinhel ASG, Paris MRP, et al. 3-Phenylcoumarin derivatives selectively modulate different steps of reactive oxygen species production by immune complex-stimulated human neutrophils. Int Immunopharmacol. 2013;15(2):387-94.

16. Sasagawa S, Kameda H, Sudo J, Tanabe $\mathrm{T}$. Inhibitory effect of nicotine on chemiluminescence response of human polymorphonuclear leukocytes stimulated by opsonized zymosan in vitro. J Toxicol Sci. 1984;9(1):1-9.

17. Sasagawa S, Suzuki K, Sakatani T, Fujikura T. Effects of Nicotine on the Functions of Human Polymorphonuclear Leukocytes in Vitro. J Leukoc Biol. 1985;37(5):493502.

18. Bernik TR, Friedman SG, Ochani M, DiRaimo R, Ulloa L, Yang H, et al. Pharmacological stimulation of the cholinergic antiinflammatory pathway. J Exp Med. 2002;195(6):781-788.

19. Tracey KJ. Understanding immunity requires more than immunology. Nat Immunol. 2010;11(7):561-564. doi: 10.1038/ni0710-561.

20. Brunskole Hummel I, Reinartz MT, Kalble S, Burhenne H, Schwede F, Buschauer A, et al. Dissociations in the effects of beta2-adrenergic receptor agonists on cAMP formation and superoxide production in human neutrophils: support for the concept of functional selectivity. PloS One. 2013;8(5):e64556. doi: 10.1371/ journal.pone.0064556.

21. Dal Sasso M, Bovio C, Culici M, Fonti E, Braga PC. The SH-metabolite I of erdosteine, a mucolytic drug, enhances the inhibitory effect of salbutamol on the respiratory burst of neutrophils. Drugs Exp Clin Res. 2002;28(4):147-54.
22. Keranen $\mathrm{T}$, Hommo $\mathrm{T}$, Hamalainen M, Moilanen E, Korhonen R. AntiInflammatory Effects of beta2-Receptor Agonists Salbutamol and Terbutaline Are Mediated by MKP-1. PLoS One. 2016;11(2):e0148144. doi: 10.1371/ journal.pone.0148144.

23. Hellstrand K, Hermodsson S, Strannegard $O$. Evidence for a beta-adrenoceptor-mediated regulation of human natural killer cells. J Immunol. 1985;134(6):4095-9.

24. Nishina K, Akamatsu H, Mikawa K, Shiga M, Maekawa N, Obara H, et al. The effects of clonidine and dexmedetomidine on human neutrophil functions. Anesth Analg. 1999;88(2): 452-8.

25. Xiao J, Huang HW, Peng YP, Bao JY, Huang Y, Qiu YH. Modulation of natural killer cell function by alpha-adrenoreceptor-coupled signalling. Neuro Endocrinol Lett. 2010;31(5):635-44.

26. Watanabe Y, Nakayama T, Nagakubo D, Hieshima K, Jin Z, Katou F, et al. Dopamine selectively induces migration and homing of naive CD8+ T cells via dopamine receptor D3. J. Immunol. 2006;176(2):848-856.

27. Leon-Ponte M, Ahern GP, O'Connell PJ. Serotonin provides an accessory signal to enhance T-cell activation by signaling through the 5-HT7 receptor. Blood. 2007;109(8):3139-3146.

28. Kawashima K, Yoshikawa K, Fujii YX, Moriwaki Y, Misawa H. Expression and function of genes encoding cholinergic components in murine immune cells. Life Sci. 2007;80(24-25):2314-2319.

Correspondencia: Job Nexar Quispe Huaman Dirección: Thunstrasse 22, Steffisburg, Suiza. Email:jobnexar@gmail.com 